\title{
An In Vitro Evaluation of Antibacterial Efficacy of Various Concentration of Eucalyptus globulus Leaf Extract on Periodontal Pathogens
}

\author{
Praveen K Bankur ${ }^{1}$, Melwin Mathew ${ }^{2}$, Sultan A Almalki ${ }^{3}$, Mohammad Jalaluddin ${ }^{4}$, Ipsita Jayanti ${ }^{5}$, Macha Durgaraju ${ }^{6}$
}

\begin{abstract}
Aim: The aim of the present study was to evaluate the antibacterial efficacy of various concentrations of Eucalyptus globulus leaf extract on periodontal pathogens.

Materials and methods: Matured, healthy and disease-free leaves of Eucalyptus globulus were collected. The leaves were washed under tap water and finally dried in an oven at a temperature of $45^{\circ} \mathrm{C}$ for 48 hours. The dried plants were ground in an electric blender to make them into a powder. The powder was mixed with $100 \%$ ethanol and kept it inside a shaker overnight at $35^{\circ} \mathrm{C}$. The mixture was centrifuged for 10 minutes at 2,500 rpm. Three different concentrations $(10 \%, 50 \%$, and $100 \% \mathrm{v} / \mathrm{v})$ were used as antibacterial agents. Chlorhexidine $(0.2 \%)$ was considered as positive control and dimethyl formamide was considered as negative control against $P$. gingivalis and A. actinomycetemcomitans. The disc diffusion method was used to determine the extract's antibacterial activity against the test organisms. A digital Vernier caliper was used to measure the diameter of antibacterial activity showing the zone of inhibition in millimeters.

Results: Eucalyptus globulus with $100 \%$ concentration showed a maximum zone of inhibition against $A$. actinomycetemcomitans and $P$. gingivalis $(5.38 \pm 0.32 \mathrm{~mm}, 4.82 \pm 0.11 \mathrm{~mm})$ followed by $50 \%$ and $10 \%$ accordingly. The negative control of dimethyl formamide showed a zone of inhibition of $0.48 \pm 0.96 \mathrm{~mm}$ and $0.63 \pm 0.20 \mathrm{~mm}$ against $A$. actinomycetemcomitans and $P$. gingivalis. The positive control of $0.2 \%$ chlorhexidine showed a zone of inhibition of $8.46 \pm 1.02 \mathrm{~mm}$ and $7.18 \pm 0.54 \mathrm{~mm}$ against $A$. actinomycetemcomitans and $P$. gingivalis. The ANOVA test showed a highly significant antibacterial efficacy in $0.2 \%$ chlorhexidine and $100 \%$ concentration Eucalyptus globulus.

Conclusion: A significant maximum zone of inhibition against A. actinomycetemcomitans and $P$. gingivalis was showed by $100 \%$ concentration of Eucalyptus globulus.

Clinical significance: Other than the systemic diseases treatment, Eucalyptus globulus also serves as an effective promising alternative to antibiotics in the prevention of oral infections because of the natural phytochemicals existing in them.

Keywords: A. actinomycetemcomitans, Eucalyptus globulus, P. gingivalis, Zone of inhibition.

The Journal of Contemporary Dental Practice (2019): 10.5005/jp-journals-10024-2639
\end{abstract}

\section{INTRODUCTION}

Clinically, dental plaque is defined as a structured, resilient, yellowgrayish material that adheres tenaciously to the intraoral hard surfaces, including fixed and removable prosthesis. Dental plaque serves as a potential etiological risk factor causing periodontal diseases and has been medically documented. The well-known method in preventing periodontitis and gingivitis is to completely remove the plaque from the dentogingival region. ${ }^{1}$

The main problem in the management of the oral infection is the resistance to the antimicrobial activity of the drugs, due to the formation of biofilm by dental plaque. There are many antimicrobial drugs available that can provide desirable clinical results in improving the condition. Still, there exists an obstruction in the success of these antimicrobial agents local delivery. The repeated use of multiple antimicrobials previously has led to the formation of bacteria resistant to multidrugs. Hence, the natural ingredients are coming to light and grabbing the researchers' attention. ${ }^{2}$

The serious threat is that the multiplication of drug-resistant pathogens hinders the success of microbial disease treatment. The plant extracts and essential oils have evoked curiosity to be used as natural sources for the treatment. The essential oils are oily aromatic liquids called volatile oils obtained from woods, herbs, twigs, roots, bark, leaves, flowers, seeds, and buds. There are 3,000 essential oils and 300 of them are famous in the fragrance market. ${ }^{3}$ \begin{tabular}{l}
\hline \hline Department of Periodontics, Al Badar Rural Dental College and \\
Hospital, Gulbarga, Karnataka, India \\
${ }^{2}$ Department of Periodontics, Melaka Manipal Medical College, \\
Melaka, Malaysia \\
${ }^{3}$ Department of Preventive Dental Sciences, College of Dentistry, \\
Prince Sattam Bin Abdulaziz University, Al-Kharj, Kingdom of Saudi \\
Arabia \\
${ }^{4,5}$ Department of Periodontics and Oral Implantology, Kalinga Institute \\
of Dental Sciences, KIIT Deemed to be University, Bhubaneswar, \\
Odisha, India \\
${ }^{6}$ Department of Prosthodontics, HSRSM Dental College and Hospital, \\
Hingoli, Maharashtra, India
\end{tabular}

Corresponding Author: Praveen KBankur, Department of Periodontics, Al Badar Rural Dental College and Hospital, Gulbarga, Karnataka, India, Phone: +91 9886542751, e-mail: praveenbankur@gmail.com

How to cite this article: Bankur PK, Mathew M, et al. An In Vitro Evaluation of Antibacterial Efficacy of Various Concentration of Eucalyptus globulus Leaf Extract on Periodontal Pathogens. J Contemp Dent Pract 2019;20(9):1041-1044.

Source of support: Nil

Conflict of interest: None

The most commonly used plant product in traditional medicine is the Eucalyptus sp. Native to Australia, Eucalyptus has nearly

o The Author(s). 2019 Open Access This article is distributed under the terms of the Creative Commons Attribution 4.0 International License (https://creativecommons. org/licenses/by-nc/4.0/), which permits unrestricted use, distribution, and non-commercial reproduction in any medium, provided you give appropriate credit to the original author(s) and the source, provide a link to the Creative Commons license, and indicate if changes were made. The Creative Commons Public Domain Dedication waiver (http://creativecommons.org/publicdomain/zero/1.0/) applies to the data made available in this article, unless otherwise stated. 
900 species and subspecies, belonging to Myrtaceae family. ${ }^{4}$ They are well known for their fast growth, some of the species are exceptionally tall and are among the tallest trees in the word, with a height ranging between 20 meters and 50 meters. ${ }^{5}$ Therefore, this study was conducted to know the antibacterial efficacy of Eucalyptus globulus leaf extract in various concentrations on periodontal pathogenic organisms.

\section{Materials and Methods}

The present study was conducted at the Department of Periodontics, Guru Gobind Singh College of Dental Sciences and Research Centre, Burhanpur, Madhya Pradesh, India.

\section{Plant Materials}

The samples of matured, healthy, and disease-free leaves of Eucalyptus globulus were collected. The leaves were washed under tap water and cleaned in the laboratory. The washed leaves were dried initially under a shade and later dried in an oven at a temperature of $45^{\circ} \mathrm{C}$ for 48 hours. The dried leaves were ground in an electric blender to make them into a powder.

\section{Preparation of Plant Extract}

The powder from the Eucalyptus globulus extract was mixed with $100 \%$ ethanol and kept it inside a shaker overnight at $35^{\circ} \mathrm{C}$. The mixture was centrifuged for 10 minutes at 2,500 rpm. The centrifuged product was filtered using a Buchner funnel and a Whatman no. 1 filter paper. The filtrate so obtained was reduced at a low temperature to obtain a solid residue of Eucalyptus globulus extract and this was stored in sample bottles at $4^{\circ} \mathrm{C}$ before use.

\section{Preparation of Different Concentrations of Eucalyptus globulus Extract}

One gram of extract was dissolved in a $10 \mathrm{~mL}$ of dimethyl formamide to get a $10 \%$ Eucalyptus globulus extract. In a similar pattern, three different concentrations (10\%, 50\% and $100 \% \mathrm{v} / \mathrm{v}$ ) were used as antibacterial agents. Chlorhexidine (0.2\%) was considered as the positive control and dimethyl formamide was considered as the negative control.

\section{Growth of Tested Microorganisms}

Kanamycin blood agar was used to isolate $P$. gingivalis. The kanamycin blood agar consisted of $100 \mathrm{mg} / \mathrm{L}$ of kanamycin, the 0020 base of trypticase blood agar with $5 \%$ sheep blood with yeast extract supplement, vitamin k1, hemin, and l-cysteine.

Dentaid agar was used to isolate A. actinomycetemcomitans. The ingredients of Dentaid agar includes brain heart infusion (BHI) agar with $5 \mathrm{~g}$ of yeast extract, $1 \mathrm{~g}$ of sodium formate, and $1.5 \mathrm{~g}$ of sodium fumarate per liter. The medium was autoclaved at $121^{\circ} \mathrm{C}$ for 15 minutes. The final $\mathrm{PH}$ of $7.2 \pm 0.2$ was estimated. The medium was further cooled till $50^{\circ} \mathrm{C}$ and a final concentration of $9 \mu \mathrm{g} / \mathrm{mL}$ by adding vancomycin.

The subculture of $P$. gingivalis and $A$. actinomycetemcomitans were prepared by incubating them for $48-72$ hours at $35-37^{\circ} \mathrm{C}$. The agar plates were inoculated and kept it in an anaerobic jar and incubated for 48 hours, which was again incubated for another 2-4 days to aid in the formation of colonies, especially for the slow-growing organisms. P. gingivalis formed as black and mucoid colonies, whereas $A$. actinomycetemcomitans formed as tiny, translucent colonies.

\section{Assessment of Antibacterial Activity}

The disk diffusion method was used to determine the extract's antibacterial activity against the test organisms. ${ }^{6}$ A sterilized and dried filter paper of diameter $6 \mathrm{~mm}$ were coated with a premeasured amount of the test substance using a micropipette and the residual solvents were allowed to evaporate completely. The disk with the test substance was kept in nutrient agar medium seeded uniformly with the test microorganisms.

Total ten test plates with 3 disks in each were taken for this study. Each plate with all three disks was coated with the same sample. Five plates were used for $P$. gingivalis and another five were used for A. actinomycetemcomitans microorganisms. These were kept at a low temperature of $4^{\circ} \mathrm{C}$ for 24 hours, allowing for a maximum dispersion of the test materials. The plates were incubated for 24 hours at $37^{\circ} \mathrm{C}$ to allow the organisms to grow maximally. A digital Vernier caliper was used to measure the zone of inhibition in millimeters. The mean values of each sample were noted down.

\section{Statistical Analysis}

The mean and standard deviation of the results were calculated and analyzed using SPSS software. A comparison between and within the samples was done using one-way analysis of variance (ANOVA). The data were proved to be significant with the $p$ value less than 0.05 .

\section{RESULT}

The mean zone of inhibition in the extract of different concentrations of Eucalyptus globulus, $0.2 \%$ chlorhexidine, and dimethyl formamide is shown in Table 1. The maximum zone of inhibition was seen in Eucalyptus globulus with $100 \%$ concentration (5.38 \pm $0.32 \mathrm{~mm}, 4.82 \pm 0.11 \mathrm{~mm}$ ) against $A$. actinomycetemcomitans and P. gingivalis, followed by $50 \%$ and $10 \%$ concentrations. The negative control of dimethyl formamide showed a zone of inhibition of $0.48 \pm 0.96 \mathrm{~mm}$ and $0.63 \pm 0.20$ and the positive control of $0.2 \%$ chlorhexidine showed a zone of inhibition of $8.46 \pm 1.02 \mathrm{~mm}$ and $7.18 \pm 0.54 \mathrm{~mm}$ against $A$. actinomycetemcomitans and $P$. gingivalis, respectively.

The ANOVA tests shows the intra- and inter-group comparisons in Table 2. The results were showed to be highly significant with $p<0.0001$.

Table 1: Mean zone of inhibition of different concentrations of Eucalyptus globulus extract, dimethyl formamide, and $0.2 \%$ chlorhexidine

\begin{tabular}{lll}
\hline $\begin{array}{l}\text { Eucalyptus globulus } \\
\text { extract }\end{array}$ & $\begin{array}{l}\text { A.actinomycetemcomitans } \\
(\mathrm{mm})\end{array}$ & P.gingivalis $(\mathrm{mm})$ \\
\hline $10 \%$ & $1.20 \pm 0.38$ & $1.12 \pm 0.41$ \\
$50 \%$ & $2.75 \pm 0.11$ & $1.98 \pm 0.86$ \\
$100 \%$ & $5.38 \pm 0.32$ & $4.82 \pm 0.11$ \\
Dimethyl formamide & $0.48 \pm 0.96$ & $0.63 \pm 0.20$ \\
$0.2 \%$ chlorhexidine & $8.46 \pm 1.02$ & $7.18 \pm 0.54$ \\
\hline
\end{tabular}

Table 2: Evaluation of zone of inhibition among Eucalyptus globulus extracts using ANOVA

\begin{tabular}{lcrll}
\hline & Sum of squares & Df & $F$ & Sig. \\
\hline Between groups & 3286.544 & 6 & 1042.133 & 0.0001 \\
Within groups & 21.108 & 36 & & \\
Total & 3307.652 & 42 & & \\
\hline
\end{tabular}


Tables 3 and 4 show the comparison between the Eucalyptus globulus extract of different concentrations, $0.2 \%$ chlorhexidine, and dimethyl formamide with $A$. actinomycetemcomitans and P. gingivalis. Chlorhexidine (0.2\%) and Eucalyptus globulus extract (100\%) displayed a statistically significant difference (Fig. 1).

\section{Discussion}

The primary causative agent for the development of the periodontal disease is dental plaque. The long-standing dental plaque, uninterrupted by oral hygiene methods may lead to gingivitis, which progress to become periodontitis. Chemotherapeutic agents and mechanical methods were commonly used to treat periodontal disease. The limitation of the chemotherapeutic agents is microbial resistance and toxicity. In order to overcome these limitations, there is a search for newer and safer chemotherapeutic agents. A natural

Table 3: Comparison between different concentrations of Eucalyptus globulus extract, dimethyl formamide, and $0.2 \%$ chlorhexidine with A. actinomycetemcomitans

\begin{tabular}{lcll}
\hline $\begin{array}{l}\text { Eucalyptus globulus } \\
\text { extract }\end{array}$ & $\begin{array}{l}\text { A.actinomycetem- } \\
\text { comitans }(\mathrm{mm})\end{array}$ & Std. error & p value \\
\hline $10 \%$ & $1.20 \pm 0.38$ & 0.1316 & 0.213 \\
$50 \%$ & $2.75 \pm 0.11$ & 0.2260 & 0.07 \\
$100 \%$ & $5.38 \pm 0.32$ & 0.1840 & 0.001 \\
Dimethyl formamide & $0.48 \pm 0.96$ & 0.2108 & 0.107 \\
$0.2 \%$ chlorhexidine & $8.46 \pm 1.02$ & 0.2432 & 0.001 \\
\hline
\end{tabular}

Table 4: Within group comparison between different concentrations of Eucalyptus globulus extract, dimethyl formamide, and $0.2 \%$ chlorhexidine with $P$. gingivalis

\begin{tabular}{lcll}
\hline $\begin{array}{l}\text { Eucalyptus globulus } \\
\text { extract }\end{array}$ & P. gingivalis $(\mathrm{mm})$ & Std. error & p value \\
\hline $10 \%$ & $1.12 \pm 0.41$ & 0.1520 & 0.12 \\
$50 \%$ & $1.98 \pm 0.86$ & 0.0322 & 0.06 \\
$100 \%$ & $4.82 \pm 0.11$ & 0.1890 & 0.001 \\
Dimethyl formamide & $0.63 \pm 0.20$ & 0.1109 & 0.81 \\
$0.2 \%$ chlorhexidine & $7.18 \pm 0.54$ & 0.1312 & 0.001 \\
\hline
\end{tabular}

phytochemical agent extracted from plants is known to be a better alternative for synthetic chemicals. ${ }^{7}$

The most common and effective anti-plaque agent commonly used is chlorhexidine mouthwash. Therefore, chlorhexidine is used as a standard comparative agent but it causes a discoloration of the tongue and teeth, a burning sensation in the oral cavity, selective mouth dryness, and can cause taste loss. Resistance to the antibacterial drugs is seen in most of the bacterial species. ${ }^{8}$

This study showed that $100 \%$ concentration of the Eucalyptus globulus extract shows a comparatively maximum inhibition zone against $P$. gingivalis and $A$. actinomycetemcomitans. The study has been done recently by Nagata et al. ${ }^{9}$ on virulence factors of Porphyromonas gingivalis, including Arg and Lys specific cysteine proteinases. A $6 \%$ ethanol extract proved effective against periodontal organisms such as P. gingivalis and Prevotella intermedia. In particular, among periodontopathic bacteria, the growth of $P$. gingivalis was strongly inhibited even with a low concentration $(10 \mathrm{mg} / \mathrm{mL})$ of eucalyptus extracts.

Hans et al. ${ }^{10}$ tested various concentrations of eucalyptus, chamomile, turmeric, and tea tree against the notorious periopathogen $P$. gingivalis. In their study, eucalyptus had the maximum antimicrobial efficacy against $P$. gingivalis.

Eucalyptus, which hails from the family Myrtaceae, has been utilized as an infection-controlling agent since ancient Egypt times. ${ }^{11}$ Eucalyptus oil's analgesic, anti-inflammatory, and antimicrobial properties have been reported by Cimanga et al. ${ }^{12}$ and Silva et al. ${ }^{13}$ The anti-microbial property of eucalyptus according to Tian et al. ${ }^{14}$ is mainly because of its potential components citronellal (about 57\%), followed by citronellol (about 16\%) and citronellyl acetate (about 15\%). However, the antimicrobial bioactivity can be attributed to a-terpineol, which showed eightfold higher activity than citronellol against Staphyllococcus aureus. Eucalyptus oil resisted the growth of periodontal pathogens (including $P$. gingivalis), with a concentration ranged from $0.25 \%$ to $0.5 \%$ in other strains. ${ }^{15}$

The study conducted by Motamayel et al. ${ }^{16}$ showed that the extract of Eucalyptus had no effect on cancer-causing bacteria such as L. acidophilus and S. mutans. The anti-cariogenic effect of eucalyptus under anti-microbial activity is not yet researched.
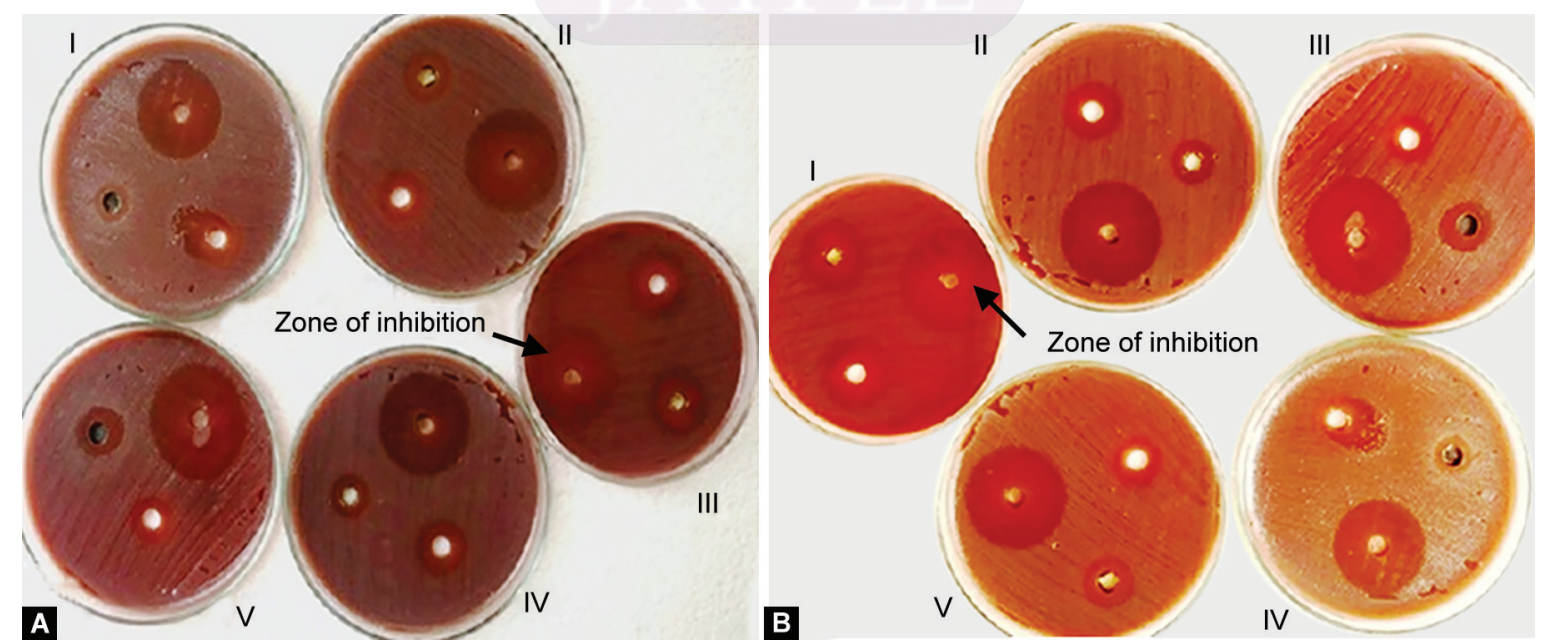

Figs $1 \mathrm{~A}$ and B: (A) Zone of inhibition of different concentrations of Eucalyptus globulus extract, dimethyl formamide, and $0.2 \%$ chlorhexidine against P. gingivalis (I. dimethyl formamide, II. 50\% extract, III. 0.2\% chlorhexidine, IV. 10\% extract, V. 100\% extract); (B) Zone of inhibition of different concentrations of Eucalyptus globulus extract, dimethyl formamide, and $0.2 \%$ chlorhexidine against Aggregatibacter actinomycetemcomitans (I. $0.2 \%$ chlorhexidine, II. 100\% extract, III. 10\% extract, IV. dimethyl formamide, V. 50\% extract) 
However, the results of previous studies proved the inhibitory activity of eucalyptus on the biofilm causing tooth decay. A doubleblind, randomized study conducted in 2008 by Nagata et al. ${ }^{17}$ assessed the eucalyptus extract's effect on caries and periodontal diseases. They also reported that eucalyptus-containing chewing gum can help in improving the gingival index and decrease the periodontal diseases compared to the control group.

Periodontitis is defined as a chronic inflammatory disease that can progress to intraoral dysbiosis, leading to continuous destruction of surrounding tissues such as connective tissue, periodontal ligaments, and alveolar bone, and if not treated, may lead to tooth loss. The colonization of microorganisms majorly appearing near the gingival sulcus at or near the marginal gingiva represents the periodontal health. There are hardly 15-20 species of bacteria that are found closely related to periodontal disease. Among them, $A$. actinomycetemcomitans and $P$. gingivalis (a prominent species from "red complex") are found to be the most virulent. The immune response induced by the intraoral dysbiotic also contributes to the periodontal disease progression, which is not always caused by a virulent bacterial invasion. ${ }^{18}$ As the periodontitis is more prevalent, the adverse effect of the anti-microbials currently used in the oral disease treatment and antibacterial resistance of bacteria are of main concern. The safe, equally effective alternative is needed for the prevention and treatment of the oral disease. Eucalyptus globulus extracts in the present study can serve as a better alternative to antibiotics.

The limitation in this study is that the combination of Eucalyptus globulus extracts enhances the antimicrobial effectiveness owing to its synergetic action instead of slowing down the bacterial resistance development. Therefore these combination extracts are more effective alternatives for chlorhexidine. Furthermore, in vivo studies on humans are much needed to validate the results of in vivo studies.

\section{Conclusion}

A significant maximum zone of inhibition against $A$. actinomycetemcomitans and P. gingivalis was showed by $100 \%$ concentration of Eucalyptus globulus.

\section{References}

1. Menon L, Ramamurthy J. New vistas in plaque control. IOSR J Dent Med Sci 2014;13:64-68. DOI: 10.9790/0853-13356468.

2. Pogue JM, Kaye KS, et al. Appropriate antimicrobial therapy in the era of multidrug-resistant human pathogens. Clin Microbiol Infect 2015;21:302-312. DOI: 10.1016/j.cmi.2014.12.025.
3. Prabuseenivasan $\mathrm{S}$, Jayakumar $\mathrm{M}$, et al. In vitro antibacterial activity of some plant essential oils. BMC Complement Altern Med 2006;6:39. DOI: 10.1186/1472-6882-6-39.

4. Pereira V, Dias C, et al. Antibacterial activity and synergistic effects between Eucalyptus globulus leaf residues (essential oils and extracts) and antibiotics against several isolates of respiratory tract infections (Pseudomonas aeruginosa). Ind Crops Prod 2014;52:1-7. DOI: 10.1016/ j.indcrop.2013.09.032.

5. Seyyed Nejad M, Motamedi H, et al. Antibacterial effect of Eucalyptus microtheca. Int J Enteric Pathog 2014;2(2):1-5.

6. Nostro AM, Germano P, et al. Extraction methods and bioautography for evaluation of medicinal plant antimicrobial activity. Lett Appl Microbiol 2000;30:379-384. DOI: 10.1046/j.1472-765x.2000.00731.x.

7. Ravi K, Divyashree P. Psidium guajava: A review on its potential as an adjunct in treating periodontal disease. Pharmacogn Rev 2014;8:96-100. DOI: 10.4103/0973-7847.134233.

8. Kshirsagar MM, Dodamani AS, et al. Antibacterial activity of garlic extract on cariogenic bacteria: An in vitro study. Ayu 2018;39:165-168. DOI: 10.4103/ayu.AYU_193_16.

9. Nagata $\mathrm{H}$, Inagaki $\mathrm{Y}$, et al. Inhibitory effects of macrocarpals on the biological activity of Porphyromonas gingivalis and other periodontopathic bacteria. Oral Microbiol Immunol 2006;21:159-163. DOI: 10.1111/j.1399-302X.2006.00269.x.

10. Hans VM, Grover HS, et al. Antimicrobial Efficacy of Various Essential Oils at Varying Concentrations against Periopathogen Porphyromonas gingivalis. J Clin Diagn Res 2016;10(9):ZC16-ZC19. DOI: 10.7860/JCDR/2016/18956.8435.

11. Luqman S, Dwivedi GR, et al. Antimicrobial activity of Eucalyptus citriodora essential oil. Int J Essen Oil Ther 2008;2:69-75.

12. Cimanga K, Kambu K, et al. Correlation between chemical composition and antibacterial activity of essential oils of some aromatic medicinal plants growing in the Democratic Republic of Congo. J Ethnopharmacol 2002;79:213-220. DOI: 10.1016/S03788741(01)00384-1.

13. Silva J, Abebe $W$, et al. Analgesic and anti-inflammatory effects of essential oils of Eucalyptus. J Ethnopharmacol 2003;89:277-283. DOI: 10.1016/j.jep.2003.09.007.

14. Tian Y, Liu X, et al. Extraction and determination of volatile constituents in leaves of Eucalyptus citriodora. Chinese J Chromatography 2005;23:651-654.

15. Takarada K, Kimizuka R, et al. A comparison of the antibacterial efficacies of essential oils against oral pathogens. Oral Microbiol Immunol 2004;19:61-64. DOI: 10.1046/j.0902-0055.2003.00111.x.

16. Motamayel FA, Hassanpour S, et al. Antibacterial effect of eucalyptus (globulus Labill) and garlic (Allium sativum) extracts on oral Cariogenic bacteria. J Microbiol Res Rev 2013;1(2):12-17.

17. Nagata $\mathrm{N}$, Inagaki Y, et al. Effect of eucalyptus extract chewing gum on periodontal health:a double-masked, randomized trial. J Periodontol 2008;79:1378-1385. DOI: 10.1902/jop.2008.070622.

18. Cekici A, Kantarci A, et al. Inflammatory and immune pathways in the pathogenesis of periodontal disease. Periodontol 2000 2014;64(1):57-80. DOI: 10.1111/prd.12002. 